International Journal of Modern Physics D

(C) World Scientific Publishing Company

DESY 11-222

\title{
COSMOLOGICAL PHASE TRANSITIONS FROM LATTICE FIELD THEORY
}

\author{
Karl Jansen \\ NIC, DESY, Platanenallee 6, 15738 Zeuthen, Germany
}

\begin{abstract}
In this proceedings contribution we discuss the fate of the electroweak and the quantum chromodynamics phase transitions relevant for the early stage of the universe at nonzero temperature. These phase transitions are related to the Higgs mechanism and the breaking of chiral symmetry, respectively. We will review that non-perturbative lattice field theory simulations show that these phase transitions actually do not occur in nature and that physical observables show a completely smooth behaviour as a function of the temperature.
\end{abstract}

Keywords: Electroweak Phase transition, QCD phase transition, Lattice Field Theory

\section{Introduction}

During its early evolution, the universe is believed to have passed through two phase transitions. The first is the electroweak phase transition at temperatures of the electroweak scale of about $250 \mathrm{GeV}$. The second is the quantum chromodynamics (QCD) phase transition at much later times and correspondingly smaller temperatures of $O(100) \mathrm{MeV}$.

Both phase transitions are associated with important phenomena related to spontaneous symmetry breaking. In case of the electroweak theory, it is the Higgs mechanism providing masses to all quarks, leptons and the weak gauge bosons. In case of QCD, it is the breaking of chiral symmetry which is an essential element in understanding of hadronic phenomena in nature such as the hadron spectrum.

Phase transitions are non-perturbative phenomena and hence need appropriate tools to be investigated theoretically. Lattice field theory is such a tool and indeed, in the past both, the electroweak and the QCD non-zero temperature phase transitions have been studied by means of numerical simulations. The picture that emerged from these simulations is rather surprising: it seems that nature has preferred to evolve the universe completely smoothly without ever passing the anticipated phase transitions described above. Thus, the universe has merely changed its state of aggregation like water does in the temperature-pressure phase diagram from the fluid (water) to the gaseous (vapor) regions of the phase diagram. In this proceedings contribution we will review the evidence that has been obtained for this picture from lattice simulations. Although the results summarized here are well known in 
large parts of the high energy physics community, they might be new and interesting to the participants of this conference which provides sufficient motivation to discuss them here.

\section{The non-zero temperature electroweak phase transition}

In the electroweak sector of the standard model our present understanding is that through the Higgs mechanism mass has been given to the electroweak gauge bosons, the quarks and the leptons. In the limit of pure scalar $\Phi^{4}$ theory, the Higgs mechanism is based on the spontaneous breaking of an $O(4)$ symmetry of the action that left behind the Higgs boson and three Goldstone bosons which then turned into massive gauge bosons through the interaction of the Higgs field when the $\mathrm{SU}(2)$ gauge fields are switched on.

The phenomenon of spontaneous symmetry breaking is associated with a phase transition and hence the universe should have undergone a non-zero temperature phase transition at which the Higgs mechanism became operative. Although the investigation of this scenario is clearly interesting in its own right, large scale simulations of the non-zero temperature electroweak phase transition were started in the context of the question, whether in the standard model of particle interactions the Sakharov conditions for explaining the baryon asymmetry of the universe can be realized. One of Sakharov's condition is that the universe had to be sufficiently out of thermal equilibrium in order that an asymmetry between baryon anti-baryon generation and annihilation processes can occur. Sufficient here means that the ratio of the scalar field expectation value $v$ over the critical temperature $T_{c}$ is larger than one, $v / T_{c}>1$. This argument can be inferred from the sphaleron transition rate and we refer to ref. 1 a for more details.

Although there were a number of first attempts within perturbation theory to clarify this question, it soon turned out that these early attempts were not very appropriate to describe the phase transition. This triggered in turn numerical simulations of the electroweak sector of the standard model both in the four dimensional system [2 5] and 3-dimensional effective theories [6,7]. In all these simulations the fermions were neglected (the value of the Yukawa coupling were set to zero) such that pure $\mathrm{SU}(2)$-Gauge-Higgs systems were considered.

From these simulations it then became possible to compute $v / T_{c}$ as a function of the Higgs boson mass $M_{\mathrm{H}}$. The results of such simulations and a comparison to a refined perturbative approach [8,9] are summarized in ref. [10] and shown in fig. 1 (left). Note that in these works which were performed in a completely gauge invariant setup the vacuum expectation value was defined as $v^{2}=\operatorname{Tr}\left\langle\Phi_{x}^{\dagger} \Phi_{x}\right\rangle$ with $\Phi_{x}$ the Higgs field. It was found that for Higgs boson masses $M_{\mathrm{H}} \lesssim 80 \mathrm{GeV}$ indeed a phase transition of first order can be established. However, for larger values of

${ }^{a}$ In this short proceedings contribution we cannot provide a comprehensive reference list. We point therefore to only selected references and reviews. 
$M_{\mathrm{H}}$ this phase transitions ends in a critical point and turns into a cross-over, see fig. 1 (right). This means that physical observables depend completely smoothly on the temperature and do not feel any phase transition. Since the present lower limit on the Higgs boson mass is $M_{\mathrm{H}}>114 \mathrm{GeV}$, as a consequence -at least within the framework of the standard model- the universe must have moved in temperature in the cross-over region and hence has not felt the presence of a phase transition. This conclusion from lattice simulations is supported by perturbative calculations of the effective potential in a gauge invariant manner up to two loops [8, 9 and from a non-perturbative renormalization group approach [11.
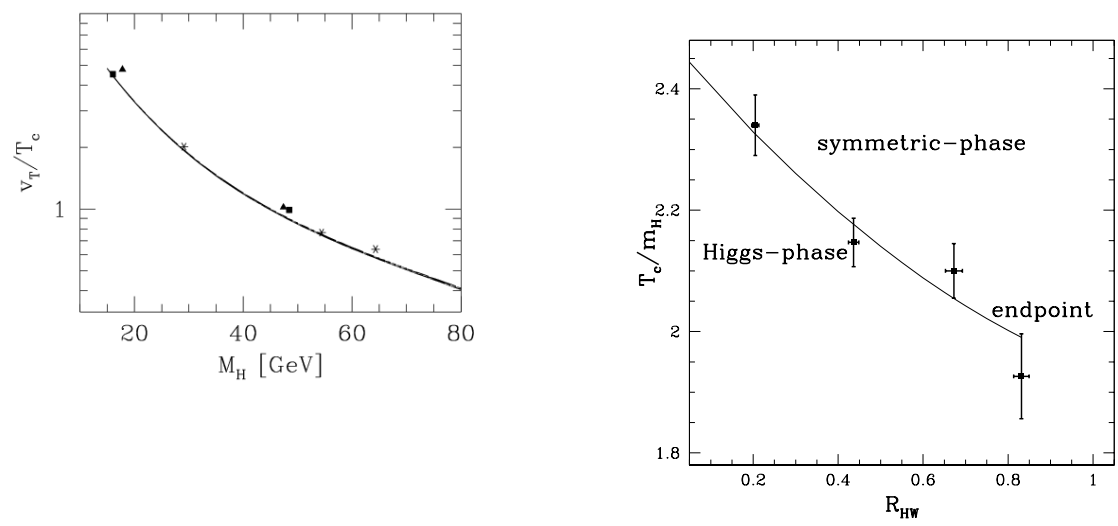

Fig. 1. Left: the ratio of the electroweak vacuum expectation value $v$ over the critical temperature $T_{c}$ is shown as a function of the Higgs boson mass. The data points are from simulations of the 4dimensional theory and effective 3 -dimensional models. The solid line is from a 2-loop perturbative analysis of the gauge invariant effective potential. The figure is taken from ref. [10]. Right: we show the ratio of the critical temperature over the Higgs boson mass as a function of the ratio $R_{\mathrm{HW}}$ of the Higgs boson over the W-boson mass. At a value of $R_{\mathrm{HW}} \approx 0.8$ the phase transition ends and turns into a crossover, demonstrating that for Higgs boson masses larger than about $100 \mathrm{GeV}$ no phase transition in the electroweak sector of the standard model has occurred.

\section{The non-zero temperature phase transition of quantum chromodynamics}

Let us now turn to the case of the strong interaction, described theoretically within quantum chromodynamics in the framework of the standard model. Here it is expected that another kind of spontaneous symmetry breaking has occurred, namely 
chiral symmetry breaking. This phenomenon in QCD is considered to be a most important aspect of the theory since it leads to the formation of a quark condensate and explains the smallness of the masses of the pions when compared to other hadron masses. The reason for this smallness is simply the association of the pions with the Goldstone bosons that emerged from the spontaneous breaking of chiral symmetry.

Again, it is expected that chiral symmetry is associated with a phase transition that occurred in the early phase of the universe at a temperature of $O(100) \mathrm{MeV}$. The determination of the critical temperature of the phase transition and its nature has been a major research direction in lattice QCD. The phase transition was first studied neglecting the quarks as dynamical degrees of freedom and indeed, a clear signal of the non-zero temperature phase transition could be established, see fig. 2 left most graph. Here, the Polyakov loop susceptibility is plotted as a function of the inverse bare gauge coupling $\beta$ corresponding to changing the temperature. The susceptibility shows a peak behaviour with an increase of the peak height and a narrowing of the peak structure when the spatial lattice size is increased. This behaviour strongly indicates that in the infinite volume limit there is indeed a phase transition.
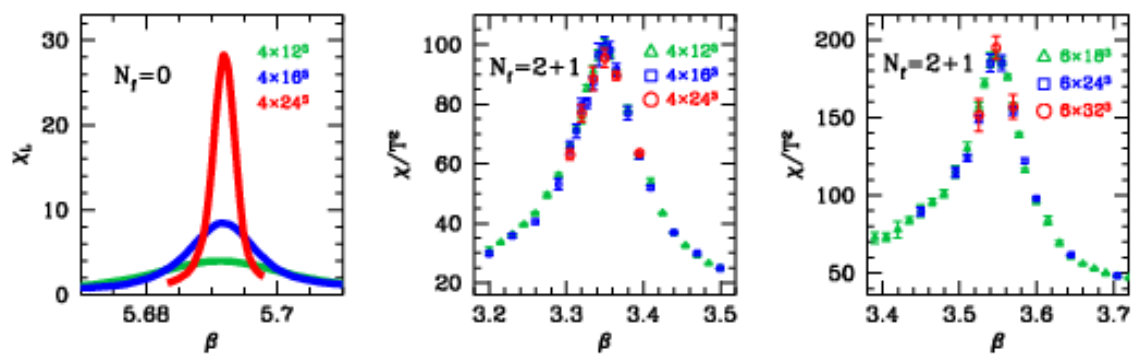

Fig. 2. We show the Polyakov loop susceptibility as a function of the temperature (encoded in the gauge coupling $\beta$ ) at fixed value of the temporal and various spatial extents of the lattice. Case (a) corresponds to the situation when quarks are not considered as dynamical degree of freedom, leftmost graph, (b) for the case of dynamical quarks with fixed time extent of the lattice $L_{t}=4$, middle and $L_{t}=6$ rightmost graph, taken from ref. [12.

When the quarks are turned on as dynamical degrees of freedom in the simulations with masses close to the physical value of the pion mass, the picture changes, however, completely. This can be seen in the middle and rightmost graphs in fig. 2 For a fixed value of the time extent of the lattice, the susceptibilities do not grow at all with increasing spatial lattice size, but fall on top of each other for all lattice 
sizes used. This means that in the infinite volume limit there is no phase transition but just a cross-over. Since the simulations were performed at about the physical value of the pion mass 13 15. this means that again all physical observables behave completely smoothly as function of the temperature and do not feel any presence of a phase transition. For other results and reviews, see ref. [16] and refs. [12, 17, respectively. Thus, nature has -as in case of the electroweak phase transition-decided to not let the universe pass through a phase transition during its evolution.

\section{Discussion}

The results of the previous sections leave us with a puzzle. In the introduction we have emphasized that the phenomenon of spontaneous symmetry breaking, associated with a phase transition, is at the heart of the standard model. It is the basis of the Higgs mechanism in the framework of the electroweak theory and chiral symmetry breaking in the case of quantum chromodynamics. The non-perturbatively obtained results from lattice field theory investigations tell us, however, that for a physical pion mass and experimentally allowed values of the Higgs boson mass the universe has not undergone any phase transition when cooling down.

In the electroweak sector of the standard model it is actually not so surprising that there is no phase transition. It is known since a long time that the symmetric regime and the broken regime of the electroweak theory are analytically connected [18 and in fact that they are one and the same phase. Indeed, for the case of zero values of the Yukawa couplings there is even an analytical proof of this statement [19.

Nevertheless, a "Higgs mechanism" is operative in the theory in the sense that one finds massive gauge bosons and Higgs boson masses. In the gauge invariant setting of lattice computations, the Higgs field $\Phi_{x}$ is unphysical and has to be replaced by local, composite and gauge invariant operators. These operators can then be used to construct suitable correlation functions from which, through a transfer matrix decomposition, the desired masses are extracted. Examples for such composite operators are $\operatorname{Tr}\left[\Phi_{x}^{\dagger} \Phi_{x}\right]$ for the Higgs boson and $\operatorname{Tr}\left[\Phi_{x}^{\dagger} U_{x, \mu} \Phi_{x+\mu} \tau\right], \tau$ being a Pauli matrix, for the case of the vector bosons. The analysis of these correlation functions provides then the spectrum of the theory [20]21] which is completely consistent with the expectation from the Higgs mechanism.

Also in the case of QCD, nature seems to have preferred a continuous behaviour of physical observables as a function of the temperature. At very high temperatures the quarks and the gluons were immersed in a plasma characteristic of the high temperature regime of QCD. When the universe evolved, at a temperature of about $O(100) \mathrm{MeV}$ the coupling grew strong, the scalar condensate increased significantly in value and the formation of bound states occurred. All this happened in a completely smooth fashion without the need of explicitly going through a phase transition, as already discussed in ref. [22. "Chiral symmetry breaking" is then operative in the sense that the observed hadron spectrum is obtained with the pions 
having much lighter masses than all other hadrons.

The purpose of this proceedings contribution has been to emphasize that in both, the electroweak and the strong sector of the standard model no phase transition occurred in the early universe. This conclusion is obtained from lattice simulations in a completely gauge invariant setup. The lessons from this observation may be the following.

- First, the Higgs mechanism of the electroweak theory and the chiral transition in QCD can both be described in a fully gauge invariant manner.

- By choosing the masses of the Higgs boson and the quarks to be large enough, in the evolution of the universe the phase transitions were avoided maybe just in order to not destroy physical phenomena, such as the baryon asymmetry, that were generated at a very early stage of the universe.

- The fact that the baryon asymmetry of the universe cannot be explained within the standard model is, of course, another manifestation of the incompleteness of the standard model neccessating its replacement by a new, so far unknown new physics.

In the end, it is however unclear, whether these observations provide non-trivial insight into the structure of the standard model, or, whether they are just the gauge invariant description of the phenomenon of spontaneous symmetry breaking.

\section{Acknowledgments}

The author thanks the organizers of SMFNS2011 in Varadero, Cuba for giving him the opportunity to discuss the topic of cosmological phase transitions from lattice simulations in a very friendly and supporting atmosphere. I am also indebted to G. Rossi and M. Testa for interesting discussions.

\section{References}

1. A. G. Cohen, D. B. Kaplan, and A. E. Nelson. Ann. Rev. Nucl. Part. Sci., 43:27-70, 1993.

2. B. Bunk, E.-M. Ilgenfritz, J. Kripfganz, and A. Schiller. Nucl. Phys., B403:453-474, 1993.

3. Z. Fodor et al. Phys. Lett., B334:405-411, 1994.

4. Z. Fodor, J. Hein, K. Jansen, A. Jaster, and I. Montvay. Nucl. Phys., B439:147-186, 1995.

5. F. Csikor, Z. Fodor, J. Hein, A. Jaster, and I. Montvay. Nucl. Phys., B474:421-445, 1996.

6. K. Kajantie, M. Laine, K. Rummukainen, and M. E. Shaposhnikov. Nucl. Phys., B466:189-258, 1996 .

7. K. Kajantie, M. Laine, K. Rummukainen, and M. E. Shaposhnikov. Phys. Rev. Lett., 77:2887-2890, 1996.

8. W. Buchmuller, Z. Fodor, and A. Hebecker. Phys. Lett., B331:131-136, 1994.

9. W. Buchmuller, Z. Fodor, and A. Hebecker. Nucl. Phys., B447:317-339, 1995.

10. K. Jansen. Nucl.Phys.Proc.Suppl., 47:196-211, 1996. 
11. B. Bergerhoff and C. Wetterich. Nucl. Phys., B440:171-188, 1995.

12. Z. Fodor and S.D. Katz, arXiv0908.3341. 2009.

13. Y. Aoki, G. Endrodi, Z. Fodor, S.D. Katz, and K.K. Szabo. Nature, 443:675-678, 2006.

14. Y. Aoki, S. Borsanyi, S. Durr, Z. Fodor, S. D. Katz, et al. JHEP, 0906:088, 2009.

15. S. Borsanyi et al. JHEP, 1009:073, 2010.

16. M. Cheng, N. H. Christ, Min Li, R. D. Mawhinney, D. Renfrew, et al. Phys.Rev., D81:054510, 2010.

17. K. Kanaya. PoS, LATTICE2010:012, 2010. * Temporary entry *.

18. E H. Fradkin and S H. Shenker. Phys.Rev., D19:3682-3697, 1979.

19. E. Seiler. Lect.Notes Phys., 159:1-192, 1982.

20. H.G. Evertz, J. Jersak, C.B. Lang, and T. Neuhaus. Phys.Lett., B171:271, 1986.

21. I. Montvay. Phys.Lett., B150:441, 1985.

22. F. Wilczek. Nature, 443:637-638, 2006. 